\title{
High-power non linear frequency converted laser diodes
}

Jensen, Ole Bjarlin; Andersen, Peter E.; Hansen, Anders Kragh; Marti, Dominik; Skovgaard, Peter M. W.; Petersen, Paul Michael

\section{Published in:}

Proceedings of SPIE

Link to article, DOI:

$10.1117 / 12.2081037$

Publication date:

2015

Document Version

Publisher's PDF, also known as Version of record

Link back to DTU Orbit

Citation (APA):

Jensen, O. B., Andersen, P. E., Hansen, A. K., Marti, D., Skovgaard, P. M. W., \& Petersen, P. M. (2015). Highpower non linear frequency converted laser diodes. In Proceedings of SPIE (Vol. 9370). [937025] SPIE International Society for Optical Engineering. Proceedings of SPIE - The International Society for Optical Engineering https://doi.org/10.1117/12.2081037

\section{General rights}

Copyright and moral rights for the publications made accessible in the public portal are retained by the authors and/or other copyright owners and it is a condition of accessing publications that users recognise and abide by the legal requirements associated with these rights.

- Users may download and print one copy of any publication from the public portal for the purpose of private study or research.

- You may not further distribute the material or use it for any profit-making activity or commercial gain

- You may freely distribute the URL identifying the publication in the public portal 


\title{
High-power non linear frequency converted laser diodes \\ Ole Bjarlin Jensen ${ }^{* a}$, Peter E. Andersen ${ }^{a}$, Anders K. Hansen ${ }^{a}$, Dominik Martia, Peter M. W. Skovgaard $^{\mathrm{b}}$ and Paul Michael Petersen ${ }^{\mathrm{a}}$ \\ ${ }^{a}$ DTU Fotonik, Department of Photonics Engineering, Technical University of Denmark, DK-4000 Roskilde, Denmark, ${ }^{b}$ Norlase ApS, Risø Huse 1, DK-4000 Roskilde, Denmark
}

\begin{abstract}
We present different methods of generating light in the blue-green spectral range by nonlinear frequency conversion of tapered diode lasers achieving state-of-the-art power levels. In the blue spectral range, we show results using single-pass second harmonic generation (SHG) as well as cavity enhanced sum frequency generation (SFG) with watt-level output powers. SHG and SFG are also demonstrated in the green spectral range as a viable method to generate up to $4 \mathrm{~W}$ output power with high efficiency using different configurations.
\end{abstract}

Keywords: tapered diode laser, second harmonic generation, sum frequency generation, visible lasers

\section{INTRODUCTION}

Many applications, e.g., within biophotonics require laser sources in the blue-green spectral range ${ }^{1}$. A preferred method for such lasers has been frequency doubled solid state lasers. Such technology tends to be bulky and expensive and therefore alternatives are desired. Diode lasers in the blue-green spectral range have emerged recently but still, the power level for diffraction limited light is limited to about $100 \mathrm{~mW}^{2,3}$. High power diode lasers are available in the near-infrared spectral range but these broad area diode lasers cannot efficiently be frequency converted to the visible spectral range.

Tapered diode lasers are an established method to generate high power in a near-diffraction limited beam at wavelengths ranging from red to infrared ${ }^{4}$. More than $10 \mathrm{~W}$ of output power in a near-diffraction limited beam has been demonstrated at wavelengths between $920 \mathrm{~nm}$ and $1064 \mathrm{~nm}^{5,6}$. Furthermore, the use of integrated Bragg gratings in such diode lasers enables narrow spectrum emission. Nonlinear frequency conversion of tapered diode lasers is an efficient method for generation of coherent light in the required blue-green spectral range ${ }^{7,8}$.

SHG of tapered diode lasers has been demonstrated at many different wavelengths and power levels ${ }^{9-12}$. Here we present the generation of up to $2.7 \mathrm{~W}$ by single-pass SHG of a single tapered diode laser. Methods for increasing this power level including SFG and cascaded SHG are shown to enable generation of up to $4 \mathrm{~W}$ at $532 \mathrm{~nm}$ in a near-diffraction limited beam. These power levels are state-of-the-art for nonlinear frequency converted diode laser systems. In addition, a method of generating visible light using SFG between a coupled cavity tapered diode laser and an external cavity tapered diode laser is also presented.

Such frequency converted laser systems are used as pump sources for Ti:sapphire lasers and examples of their use within biophotonics imaging are presented. The low noise of the diode laser sources provides enhanced image contrasts, which is important for diagnostics.

\section{SECOND HARMONIC GENERATION}

\subsection{Experimental setup}

Tapered diode lasers are a special kind of diode lasers consisting of a single-mode ridge section and a tapered amplifier section $^{13}$. The single-mode section provides good beam quality and the tapered section amplifies the output from the single-mode section yielding a high output power. The opening angle of the tapered amplifier section is chosen to suit the diffraction angle of the beam exiting the single-mode ridge section. For wavelength control, different Bragg grating structures may be integrated in the single-mode section. A sketch of a tapered diode laser is shown in Figure 1.

*ojen@fotonik.dtu.dk; phone +45 46774553; fax +45 46774588; www.fotonik.dtu.dk

Quantum Sensing and Nanophotonic Devices XII, edited by Manijeh Razeghi, Eric Tournié, Gail J. Brown, Proc. of SPIE Vol. 9370, 937025 - (C) 2015 SPIE · CCC code: 0277-786X/15/\$18 - doi: 10.1117/12.2081037 


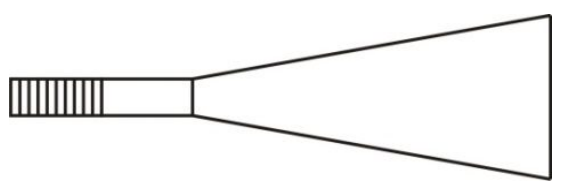

Figure 1. Sketch of a tapered diode laser. In the ridge section a Bragg grating can be included for frequency selectivity.

Single-pass SHG is the simplest way of converting near-infrared light to visible light. A sketch of the setup is shown in Figure 2. Light from the tapered diode laser is collimated in both the fast and slow axes using a combination of an aspherical lens and a cylindrical lens. A proper choice of these lenses can lead to an almost circular beam without astigmatism. Astigmatism is a challenge when using tapered diode lasers as the astigmatism depends on both the operating power and the length of the tapered section.

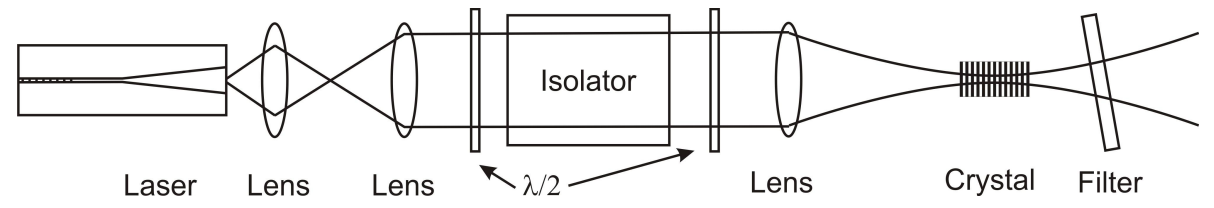

Figure 2. Sketch of a typical experimental setup for single-pass SHG of a tapered diode laser.

An optical isolator is typically used to protect the tapered diode laser from optical feedback as this can cause the laser to become unstable or even permanently damage the laser. Using a half wave plate in front of the isolator enables variation of the input power to the nonlinear crystal without changing the operating conditions and thus the astigmatism of the tapered diode laser. The light is focused inside the nonlinear crystal in order to optimize the conversion efficiency according to Boyd and Kleinman ${ }^{14}$. After the crystal, the remaining infrared light is filtered away and the frequency converted beam may be collimated to ease integration in applications.

\subsection{Generation of $532 \mathrm{~nm}$ light}

In the green wavelength range, $532 \mathrm{~nm}$ is the wavelength of most commercial solid state lasers based on the $1064 \mathrm{~nm}$ transition in Nd:YAG or Nd:YVO . Tapered diode lasers emitting at $1064 \mathrm{~nm}$ have been demonstrated with power levels above $12 \mathrm{~W}$ and with excellent spectral and spatial properties ${ }^{6}$. Single-pass frequency doubling of such lasers in $30 \mathrm{~mm}$ long PPMgLN crystals have previously been shown to generate more than $1.5 \mathrm{~W}$ of output power in a near-diffraction limited beam ${ }^{15}$. Here we have optimized the setup and have achieved up to $2.72 \mathrm{~W}$ of output power using a $50 \mathrm{~mm}$ long PPMgLN crystal in a setup similar to Figure 2. The $6 \mathrm{~mm}$ long DBR tapered diode laser was similar to the ones demonstrated $\mathrm{in}^{4,6,15}$. The light emitted by the laser was collimated in the fast axis using an 8 mm focal length aspherical lens and a cylindrical lens with $80 \mathrm{~mm}$ focal length was used to collimate the light in the slow axis and compensate for the astigmatism. After passing an optical isolator and two half wave plates, the light was focused inside the $50 \mathrm{~mm}$ long periodically poled $\mathrm{MgO}$-doped $\mathrm{LiNbO}_{3}(\mathrm{PPMgLN})$ crystal (HC Photonics). The crystal was poled with a period of 6.92 $\mu \mathrm{m}$, anti-reflection coated on both facets at both the fundamental and second harmonic wavelengths and temperature stabilized in an oven to approximately $40^{\circ} \mathrm{C}$. The laser was operated at a temperature of $20^{\circ} \mathrm{C}$ and with currents to the ridge section and taper section of $350 \mathrm{~mA}$ and $16 \mathrm{~A}$, respectively. The measured power characteristics of the SHG experiment is shown in Figure 3. The experimental results are fitted to the theoretical curve for SHG with pump depletion using the relation

$$
P_{\mathrm{SHG}}=P_{\text {laser }} \tanh ^{2}\left(\sqrt{\eta_{\text {SHG }} P_{\text {laser }}}\right)
$$

Here $\eta_{S H G}$ is the nonlinear conversion efficiency and $P_{\text {laser }}$ is the fundamental laser power. The fit gives a nonlinear conversion efficiency of $\eta_{S H G}=3.6 \% / \mathrm{W}$. The power conversion efficiency in the SHG experiment is $28.4 \%$.

The SHG beam is close to being diffraction limited with a beam quality of $\mathrm{M}^{2}<1.3$ and the spectral linewidth of the green light is smaller than $2 \mathrm{pm}$, limited by the resolution of the optical spectrum analyzer (Advantest Q8347). 


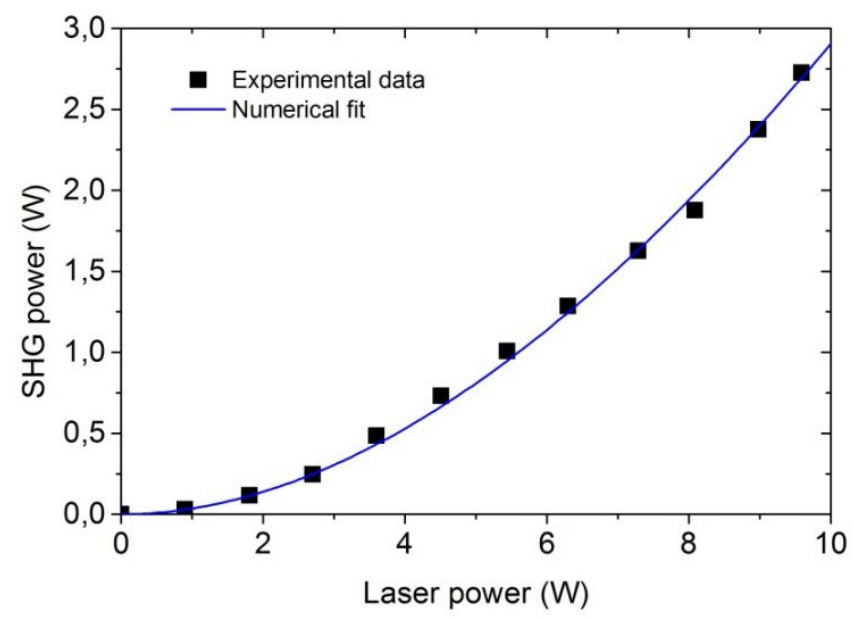

Figure 3. Measured SHG output power vs. fundamental input power. The squares are measured values and the solid curve is a fit to the experimental values using the depleted pump approximation in (1).

Such frequency doubled tapered diode lasers have been built into compact and robust laser modules. Using a $30 \mathrm{~mm}$ long PPMgLN crystal, up to $2.3 \mathrm{~W}$ of output power at $532 \mathrm{~nm}$ has been obtained. Using photodiode feedback, the output power can be stabilized to within $\pm 0.2 \%$ over extended periods of operation. An example of such a compact laser module emitting green light at $532 \mathrm{~nm}$ is shown in Figure 4.

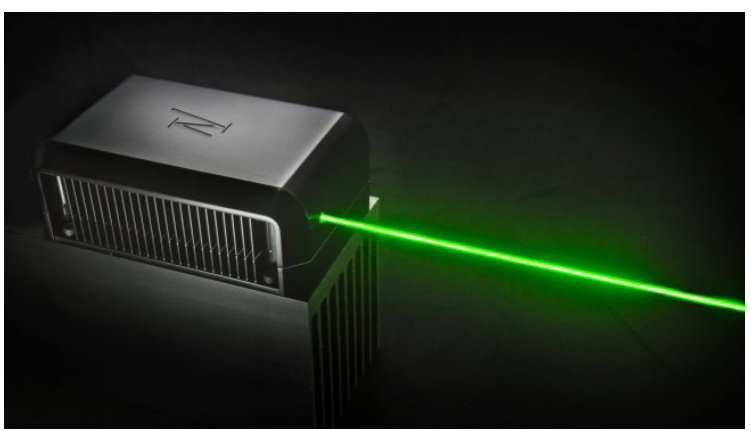

Figure 4. Compact laser module emitting more than $2 \mathrm{~W}$ of green light at $532 \mathrm{~nm}$ (Photo courtesy of Henrik Petit).

Using this simple technology, many wavelengths in the visible spectral range can be reached. Tapered diode lasers are available at many wavelengths in the near-infrared spectral region and frequency doubling of these lasers enables generation of light throughout the visible spectral region. High power has been generated at blue wavelengths at for example $460 \mathrm{~nm}$ and $488 \mathrm{~nm}$ and recently we have also demonstrated high output power at $515 \mathrm{~nm}$. Tuning of tapered lasers is possible by different means and large tuning ranges can be reached ${ }^{16-18}$. In this way tunable visible radiation can be obtained.

\subsection{Cascaded SHG setup}

In order to increase the second harmonic output power and conversion efficiency available from a single tapered diode laser, different possibilities exist. One approach is to resonate the laser beam in an enhancement cavity containing the nonlinear crystal and in this way increase the efficiency ${ }^{19}$. The complexity of this approach is, however, significant as the cavity must be held resonant with the laser frequency. A second possibility is to have multiple passes in the nonlinear crystal $^{20}$. At the power levels used in this work, heating due to absorption in the nonlinear crystal limits the efficiency as the phase matching set-point temperature for two different passes will be different. A third approach, which we exploit here, is to use a cascade of nonlinear crystals ${ }^{21}$. A sketch of the setup is shown in Figure 5. Both the fundamental and second harmonic light exiting the first nonlinear crystal is re-focused into the second nonlinear crystal. In this way, it is possible to increase the efficiency by a factor of 4 assuming no depletion of the fundamental beam. Dispersion between the fundamental and second harmonic beams necessitates compensation of the relative phase at the input to the second 
nonlinear crystal in order for the generated green light to add constructively. Here we used a plane glass plate to compensate the dispersion.

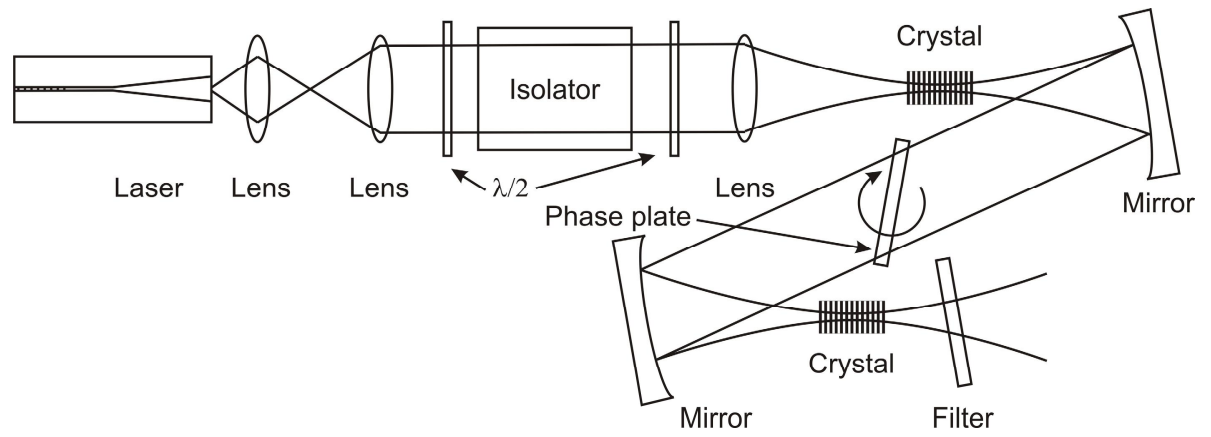

Figure 5. Schematic of the setup for cascaded SHG of a tapered diode laser using two nonlinear crystals.

Using two $30 \mathrm{~mm}$ long PPMgLN crystals, we have generated up to $3.5 \mathrm{~W}$ of light at $532 \mathrm{~nm}$ from $10 \mathrm{~W}$ of input power from the DBR tapered diode laser ${ }^{7}$. This corresponds to a factor of 2.3 more than the second harmonic power obtained from either only the first nonlinear crystal or only the second nonlinear crystal.

\section{SUM FREQUENCY GENERATION}

\subsection{Single-pass SFG setup}

In SFG the beams from two different lasers are combined and focused into a nonlinear crystal to efficiently generate a beam at the sum frequency of the two input beams. The two different lasers beams can be combined in different ways, depending on the spectral difference between the two fundamental laser beams. If the two beams have a large wavelength separation, a dichroic mirror can be used to combine the two beams. If the two lasers are closely spaced spectrally, different means than a dichroic mirror have to be used, for example a volume Bragg grating. A generic sketch of a setup for single-pass SFG with a dichroic mirror used as beam combiner is shown in Figure 6.

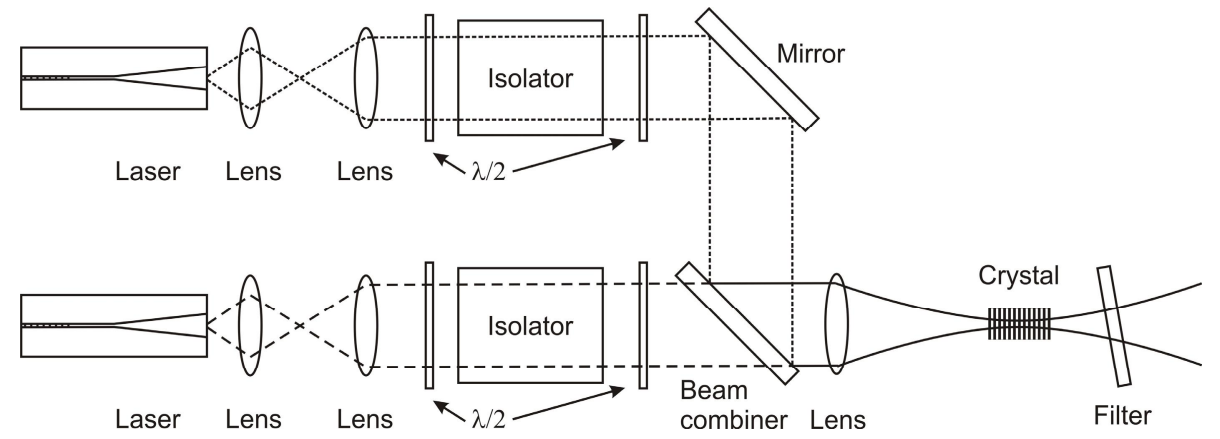

Figure 6. Sketch of experimental setup for SFG between two tapered diode lasers.

If the wavelength separation is large, two different optical isolators must be used, while a single isolator is sufficient if the lasers are within the acceptance bandwidth of the optical isolator. By combining a proper combination of tapered diode lasers, a large span of wavelengths may be reached.

\subsection{Generation of $509 \mathrm{~nm}$ light}

An experimental demonstration of SFG between two different tapered diode lasers was carried out using DBR tapered diode lasers at $978 \mathrm{~nm}$ and $1063 \mathrm{~nm}$, respectively ${ }^{22}$. Both lasers were collimated in the fast and slow axes using a combination of an aspherical lens and a cylindrical lens. After passing a combination of half wave plates and an optical isolator, the lasers were combined using a dichroic mirror. A lens focused the combined beams to a beam radius of approximately $35 \mu \mathrm{m}$ in the $20 \mathrm{~mm}$ long PPMgLN crystal (Covesion) and a filter was used to separate the generated beam at $509 \mathrm{~nm}$ from the two fundamental beams. We used a poling period in the PPMgLN crystal of $6.03 \mu \mathrm{m}$ and the crystal was temperature stabilized at $62.95^{\circ} \mathrm{C}$ for optimum phase matching. Using this setup, up to $1.73 \mathrm{~W}$ of output power was generated at a center wavelength of $509.64 \mathrm{~nm}$. The power characteristics are shown in Figure 7 . 


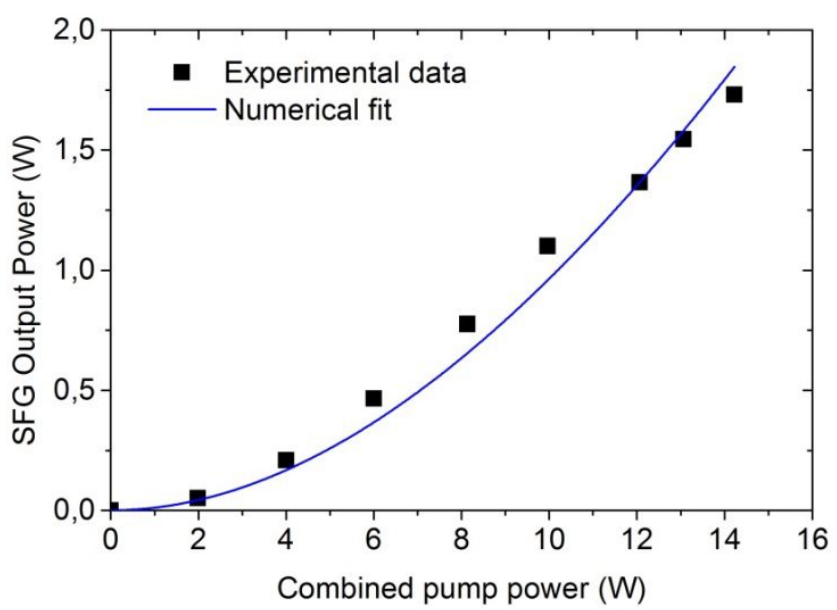

Figure 7. Measured output power at $509 \mathrm{~nm}$ vs. combined pump power. The squares illustrate the measured power values and the solid curve is a numerical fit to the experimental results using the depleted pump approximation in (1).

The $509 \mathrm{~nm}$ light was nearly diffraction limited with measured M2 values of $1.1 \mathrm{in}$ both the fast and the slow axes. The spectral linewidth of the $509 \mathrm{~nm}$ light was below $2 \mathrm{pm}$ limited by the resolution of the optical spectrum analyzer.

\subsection{Generation of $532 \mathrm{~nm}$ light}

Sum frequency generation between two nearly identical lasers was demonstrated by combining two $1063 \mathrm{~nm}$ DBR tapered lasers in a volume Bragg grating (VBG) and mixing the combined beam in a nonlinear crystal ${ }^{23}$. The two lasers were temperature tuned to have a $1 \mathrm{~nm}$ wavelength separation to allow for efficient combination in the VBG. The wavelength of the laser diffracted in the VBG must be chosen carefully to fit with the VBG characteristics and the incidence angle on the VBG. After collimation, the lasers were combined and the combined beam was sent through a combination of half wave plates and an optical isolator before being focused into a temperature stabilized $30 \mathrm{~mm}$ long PPMgLN crystal. Up to $3.9 \mathrm{~W}$ of output power at $531.5 \mathrm{~nm}$ was generated as shown in Figure 8. This corresponds to a 2.5 times increase in output power compared to SHG of a single laser. At low power a 3.2 times increase was observed. In theory, an increase by a factor of 4 could be expected at low power without pump depletion. The discrepancy can be explained by the non-ideal beam properties of the tapered diode.

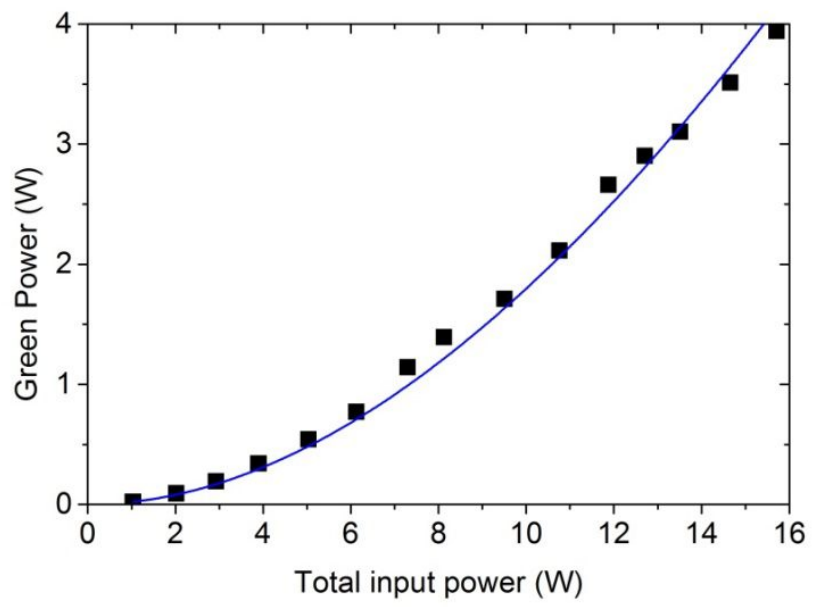

Figure 8. Measured green output power from SFG between two DBR tapered diode lasers at $1063 \mathrm{~nm}$. The squares are the experimentally obtained values and the solid curve is a numerical fit to the experimental values using the depleted pump approximation in (1).

The generated light was nearly diffraction limited with measured $\mathrm{M}^{2}$ values of $<1.1$ in the fast axis and $<1.3$ in the slow axis. The linewidth of the $532 \mathrm{~nm}$ light was below $2 \mathrm{pm}$ limited by the resolution of the optical spectrum analyzer. 


\subsection{Cavity enhanced SFG}

A different approach for the generation of visible light by SFG between two tapered diode lasers is to use a coupled ring cavity as in the sketch shown in Figure $9^{24}$. One tapered amplifier is operated in a unidirectional ring cavity which is coupled to a bow-tie enhancement cavity. This tapered amplifier operates at a single frequency dictated by the enhancement cavity resonance and the diffraction grating. The light circulating in this coupled ring cavity is automatically filtered spatially to a pure Gaussian beam due to the enhancement cavity. The circulating power in the enhancement cavity is significantly higher than the output power from the tapered amplifier and the nonlinear crystal is positioned in this cavity to take advantage of the high circulating power. A beam from an external cavity tapered diode laser is single-passed through the nonlinear crystal to generate light at the sum frequency. The generated light has a significantly better beam quality than the light emitted from the external cavity tapered diode laser due to nonlinear beam clean-up in the SFG process with the Gaussian beam inside the nonlinear crystal ${ }^{25}$.

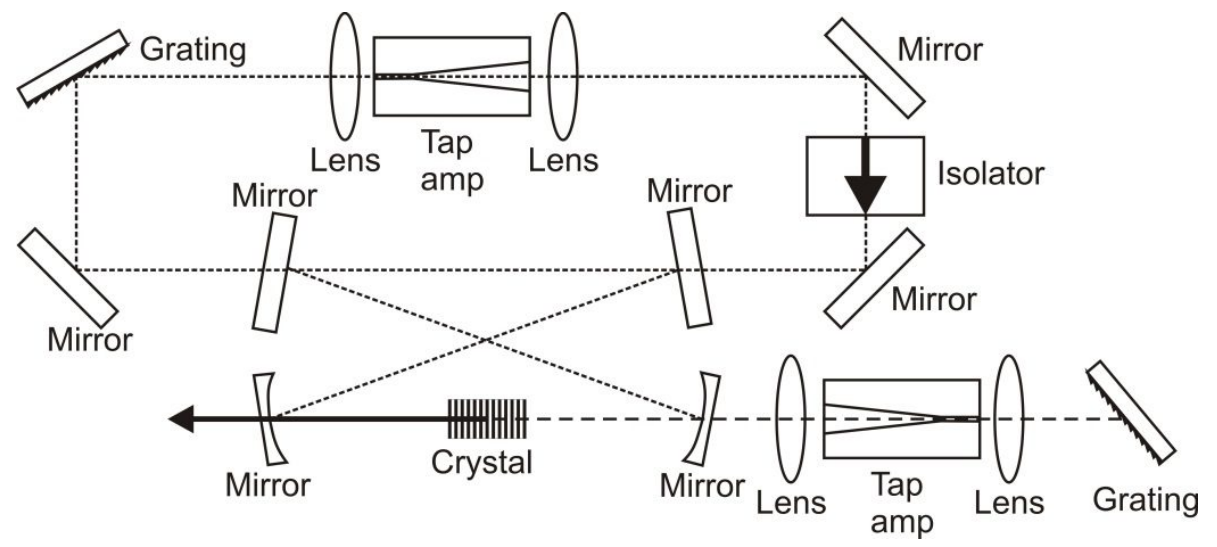

Figure 9. Simplified experimental setup for coupled ring cavity SFG between two tapered diode lasers.

Using a tapered amplifier with a center wavelength of $1060 \mathrm{~nm}$ in the coupled ring cavity and an $808 \mathrm{~nm}$ external cavity tapered laser, we have extracted $340 \mathrm{~mW}$ of blue light at $459 \mathrm{~nm}$ as shown in Figure 10. Also shown in Figure 10 is the circulating power inside the enhancement cavity. The circulating power is seen to drop when the input power at $808 \mathrm{~nm}$ is increased. At increased $808 \mathrm{~nm}$ input power, the losses from the SFG increases thus limiting the circulating power. The $459 \mathrm{~nm}$ light is nearly diffraction limited with $\mathrm{M}^{2}<1.15$ and the light is single-frequency with a linewidth below $20 \mathrm{MHz}$.

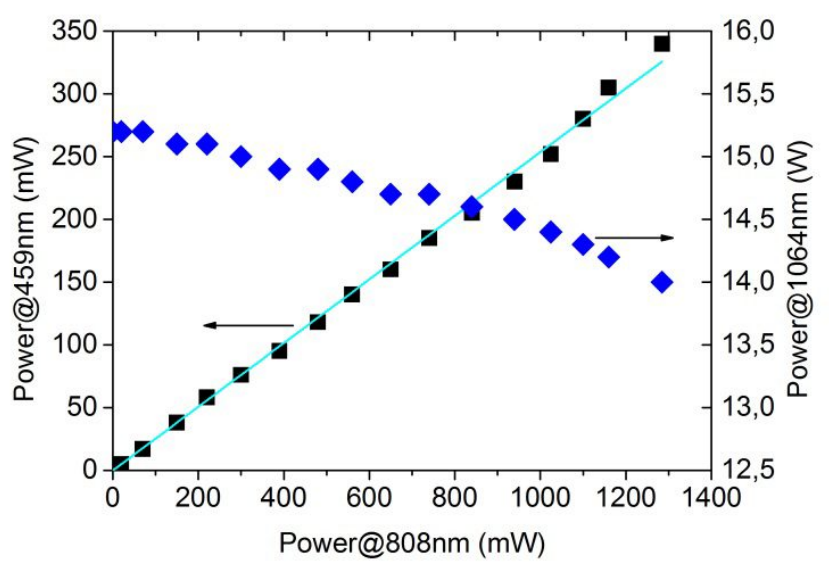

Figure 10. $459 \mathrm{~nm}$ output power vs. input power at $808 \mathrm{~nm}$ (squares). Also shown is the circulating power in the enhancement cavity (diamonds).

The $459 \mathrm{~nm}$ light can be tuned by $1.5 \mathrm{~nm}$ limited by the nonlinear crystal. A different choice of nonlinear crystal can extend the tuning range to approximately $17 \mathrm{~nm}$ for this particular choice of tapered amplifiers. Selecting different 
tapered amplifiers can extend the wavelength coverage to cover most of the visible wavelength range. A similar approach has been demonstrated for SHG of a single tapered laser in a coupled ring cavity ${ }^{26,27}$.

\section{APPLICATIONS}

\subsection{Ti:sapphire pumping}

Such frequency converted high power tapered diode laser as described above have great application potentials, for instance for pumping of Ti:sapphire lasers. Ti:sapphire lasers are typically pumped by frequency doubled solid state lasers. These lasers tend to increase the dimensions and cost of Ti:sapphire laser systems. We have used different frequency converted tapered diode lasers at $509 \mathrm{~nm}$ and $531 \mathrm{~nm}$ to pump Ti:sapphire lasers and have obtained modelocked operation with more than $180 \mathrm{~mW}$ output power and spectral widths of up to $184 \mathrm{~nm}$ (FWHM) as shown in Figure $11^{22,28}$. The pulse widths obtained have been shorter than $10 \mathrm{fs}$.

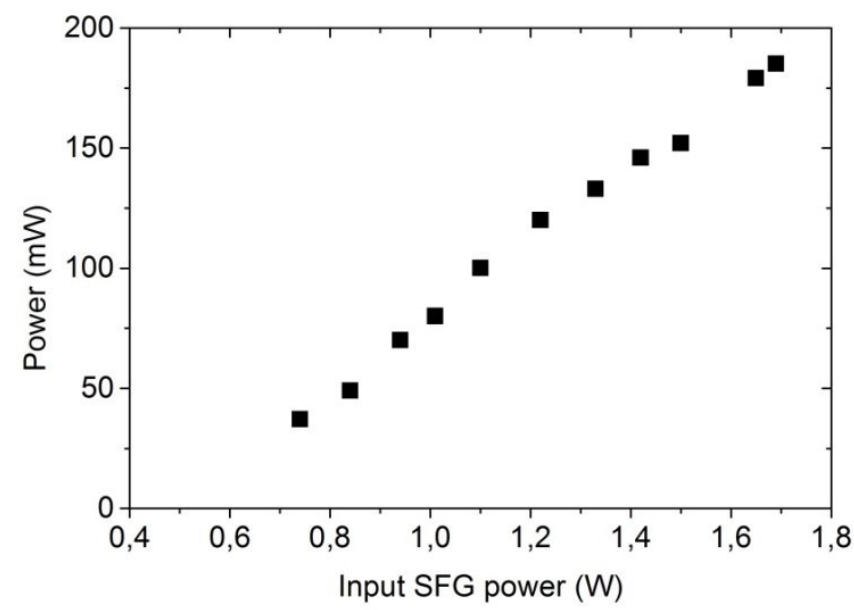

Figure 11. Mode-locked output power vs. input power at $509 \mathrm{~nm}$ from a Ti:sapphire laser.

\subsection{Optical coherence tomography}

Broadband light sources such as ultrafast Ti:sapphire lasers have shown great potential for biophotonic applications like optical coherence tomography (OCT). We have used a Ti:sapphire laser pumped by a frequency doubled tapered diode laser for retinal $\mathrm{OCT}^{29}$. The OCT system was a modified Spectralis OCT device (Heidelberg-Engineering) with built-in fixation target and eye-tracking device. The mode-locked Ti:sapphire laser was modified to emit light with a spectral width of $90 \mathrm{~nm}$ (FWHM). Only $0.8 \mathrm{~mW}$ was used for the retinal OCT, well below the safety limits. Example OCT tomograms of the retina is shown in Figure 12. The high axial resolution and good contrast in the OCT images illustrate the potential of using frequency converted tapered diode lasers for biophotonics imaging.

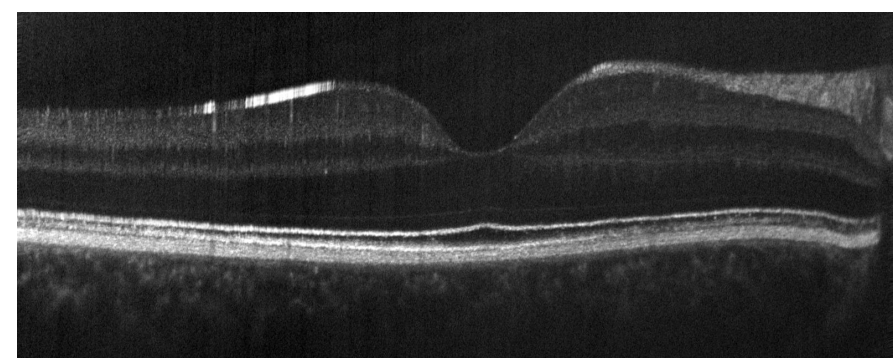

Figure 12. Measured OCT tomogram of the retina using a Ti:sapphire laser pumped by a frequency doubled tapered diode laser. 


\section{CONCLUSION}

Nonlinear frequency conversion of tapered diode lasers enables generation of several watts of power in the visible spectral region. We have shown state-of-the-art power levels for nonlinear frequency converted diode lasers. With single-pass SHG, we have generated up to $2.7 \mathrm{~W}$ of output power. Scaling this power level towards $4 \mathrm{~W}$ has been demonstrated with cascaded SHG of a single tapered diode laser and SFG between two tapered diode lasers. The excellent beam properties and narrow spectral emission enable use of such lasers in demanding applications such as pumping of Ti:sapphire lasers. Such lasers have proven excellent performance in biophotonic imaging.

The use of tapered diode lasers combined with nonlinear frequency conversion allows generation of light throughout the visible spectral range. In this way, the laser emission may be chosen to fit the desired wavelength optimal for the application.

\section{REFERENCES}

[1] Müller, A., Marschall, S., Jensen, O.B., Fricke, J., Wenzel, H., Sumpf, B., and Andersen, P.E., "Diode laser based light sources for biomedical applications," Laser \& Photonics Reviews 7(5), 605-627 (2013).

[2] Sizov, D., Bhat, R., and Zah, C., "Gallium Indium Nitride-Based Green Lasers," Journal of Lightwave Technology 30(5), 679-699 (2012).

[3] Takagi, S., Enya, Y., Kyono, T., Adachi, M., Yoshizumi, Y., Sumitomo, T., Yamanaka, Y., Kumano, T., Tokuyama, S., Sumiyoshi, K., Saga, N., Ueno, M., Katayama, K., Ikegami, T., Nakamura, T., Yanashima, K., Nakajima, H., Tasai, K., Naganuma, K., Fuutagawa, N., Takiguchi, Y., Hamaguchi, T., and Ikeda, M., "High-Power (over $100 \mathrm{~mW}$ ) Green Laser Diodes on Semipolar $\{2021\}$ GaN Substrates Operating at Wavelengths beyond 530 nm,” Applied Physics Express 5, 082102-1-3 (2012).

[4] Sumpf, B., Hasler, K.-H., Adamiec, P., Bugge, F., Dittmar, F., Fricke, J., Wenzel, H., Zorn, M., Erbert, G., and Tränkle, G., "High-Brightness Quantum Well Tapered Lasers," IEEE Journal of Selected Topics in Quantum Electronics 15(3), 1009-1020 (2009).

[5] Fiebig, C., Blume, G., Uebernickel, M., Feise, D., Kaspari, C., Paschke, K., Fricke, J., Wenzel, H., and Erbert, G., "High-Power DBR-Tapered Laser at $980 \mathrm{~nm}$ for Single-Path Second Harmonic Generation," IEEE Journal of Selected Topics in Quantum Electronics 15(3), 978-983 (2009).

[6] Sumpf, B., Hasler, K.-H., Adamiec, P., Bugge, F., Fricke, J., Ressel, P., Wenzel, H., Erbert, G., and Tränkle, G., "1060 nm DBR tapered lasers with $12 \mathrm{~W}$ output power and a nearly diffraction limited beam quality," in Proc. SPIE 7230, A. A. Belyanin and P. M. Smowton, Eds., 72301E-72301E-8 (2009).

[7] Jensen, O.B., Hansen, A.K., Müller, A., Sumpf, B., Unterhuber, A., Drexler, W., Petersen, P.M., and Andersen, P.E., "Power Scaling of Nonlinear Frequency Converted Tapered Diode Lasers for Biophotonics," IEEE Journal of Selected Topics in Quantum Electronics 20(2), 7100515 (2014).

[8] Jechow, A., Menzel, R., Paschke, K., and Erbert, G., "Blue-green light generation using high brilliance edge emitting diode lasers," Laser \& Photonics Reviews 4(5), 633-655 (2010).

[9] Waarts, R., Sanders, S., Parke, R., Mehuys, D., Lang, R., O’Brien, S., Dzurko, K., Welch, D., and Scifres, D., "Frequency-doubled monolithic master oscillator power amplifier laser diode," IEEE Photonics Technology Letters 5(10), 1122-1125 (1993).

[10] Goldberg, L., Busse, L.E., and Mehuys, D., "High power continuous wave blue light generation in KNbO3 using semiconductor amplifier seeded by a laser diode,” Applied Physics Letters 63(17), 2327-2329 (1993).

[11] Fluck, D., Pliska, T., and Günter, P., "Compact $10 \mathrm{~mW}$ all solid state $491 \mathrm{~nm}$ laser based on frequency doubling a MOPA laser diode," Optics Communications 123, 624-628 (1996).

[12] Maiwald, M., Schwertfeger, S., Güther, R., Sumpf, B., Paschke, K., Dzionk, C., Erbert, G., and Tränkle, G., “600 $\mathrm{mW}$ optical output power at $488 \mathrm{~nm}$ by use of a high-power hybrid laser diode system and a periodically poled MgO:LiNbO3 bulk crystal.," Optics Letters 31(6), 802-804 (2006).

[13] Walpole, J.N., "Semiconductor amplifiers and lasers with tapered gain regions," Optical and Quantum Electronics 28, 623-645 (1996).

[14] Boyd, G.D., and Kleinman, D.A., "Parametric interaction of focused Gaussian light beams," Journal of Applied Physics 39(8), 3597-3639 (1968). 
[15] Jensen, O.B., Andersen, P.E., Sumpf, B., Hasler, K.-H., Erbert, G., and Petersen, P.M., "1.5 W green light generation by single-pass second harmonic generation of a single-frequency tapered diode laser.," Optics Express 17(8), 6532-6539 (2009).

[16] Jensen, O.B., Sumpf, B., Erbert, G., and Petersen, P.M., "Widely Tunable High-Power Tapered Diode Laser at 1060 nm," IEEE Photonics Technology Letters 23(21), 1624-1626 (2011).

[17] Chi, M., Jensen, O.B., Holm, J., Pedersen, C., Andersen, P.E., Erbert, G., Sumpf, B., and Petersen, P.M., "Tunable high-power narrow-linewidth semiconductor laser based on an external-cavity tapered amplifier," Optics Express 13(26), 10589-10596 (2005).

[18] Kelemen, M.T., Weber, J., Rinner, F., Rogg, J., Mikulla, M., and Weimann, G., "High-brightness 1040 nm tapered diode laser," Proceedings of SPIE 4947, 252-260 (2003).

[19] Ashkin, A., Boyd, G.D., and Dziedzic, J.M., "Resonant Optical Second Harmonic Generation and Mixing," IEEE Journal of Quantum Electronics 2(6), 109-124 (1966).

[20] Spiekermann, S., Laurell, F., Pasiskevicius, V., Karlsson, H., and Freitag, I., “Optimizing non-resonant frequency conversion in periodically poled media," Applied Physics B 79(2), 211-219 (2004).

[21] Fluck, D., and Günter, P., "Efficient second-harmonic generation by lens wave-guiding in KNbO3 crystals," Optics Communications 147(4-6), 305-308 (1998).

[22] Tawfieq, M., Jensen, O.B., Hansen, A.K., Sumpf, B., Paschke, K., and Andersen, P.E., "Efficient generation of 509 nm light by sum-frequency mixing between two tapered diode lasers," Optics Communications, In press (2014), DOI: $10.1016 /$ j.optcom.2014.11.078.

[23] Müller, A., Jensen, O.B., Hasler, K.-H., Sumpf, B., Erbert, G., Andersen, P.E., and Petersen, P.M., "Efficient concept for generation of diffraction-limited green light by sum-frequency generation of spectrally combined tapered diode lasers.," Optics Letters 37(18), 3753-3755 (2012).

[24] Jensen, O.B., and Petersen, P.M., "Single-frequency blue light generation by single-pass sum-frequency generation in a coupled ring cavity tapered laser," Applied Physics Letters 103, 141107 (2013).

[25] Karamehmedović, E., Pedersen, C., Jensen, O.B., and Tidemand-Lichtenberg, P., "Nonlinear beam clean-up using resonantly enhanced sum-frequency mixing," Applied Physics B 96(2-3), 409-413 (2009).

[26] Skoczowsky, D., Jechow, A., Menzel, R., Paschke, K., and Erbert, G., "Efficient second-harmonic generation using a semiconductor tapered amplifier in a coupled ring-resonator geometry.," Optics Letters 35(2), 232-234 (2010).

[27] Jensen, O.B., and Petersen, P.M., "Generation of single-frequency tunable green light in a coupled ring tapered diode laser cavity," Optics Express 21(5), 6076-6081 (2013).

[28] Müller, A., Jensen, O.B., Unterhuber, A., Le, T., Stingl, A., Hasler, K.-H., Sumpf, B., Erbert, G., Andersen, P.E., and Petersen, P.M., "Frequency-doubled DBR-tapered diode laser for direct pumping of Ti:sapphire lasers generating sub-20 fs pulses.," Optics Express 19(13), 12156-12163 (2011).

[29] Unterhuber, A., Považay, B., Müller, A., Jensen, O.B., Duelk, M., Le, T., Petersen, P.M., Velez, C., Esmaeelpour, M., Andersen, P.E., and Drexler, W., "Simultaneous dual wavelength eye-tracked ultrahigh resolution retinal and choroidal optical coherence tomography.," Optics Letters 38(21), 4312-4315 (2013). 